\title{
Effect of miR-146a polymorphism on biochemical recurrence risk after radical prostatectomy in southern Chinese population
}

\author{
M. Chen ${ }^{1,2}$, Z.Y. Zhou ${ }^{3}$, J.G. Chen ${ }^{4}$, N. Tong ${ }^{5}$, S.Q. Chen ${ }^{1}$, Y. Yang ${ }^{1}$, \\ X.W. Zhang' ${ }^{1}$ H. Jiang ${ }^{1}$, N. Liu ${ }^{1}$, J. Liu ${ }^{1}$, G.Z. Sha ${ }^{1}$, W.D. Zhu ${ }^{1}$, \\ L.X. Hua ${ }^{6}$, Z.J. Wang ${ }^{6}$ and B. Xu ${ }^{1,2}$ \\ ${ }^{1}$ Department of Urology, Affiliated Zhongda Hospital of Southeast University, \\ Nanjing, China \\ ${ }^{2}$ Surgery Research Center, Medical School of Southeast University, \\ Nanjing, China \\ ${ }^{3}$ Department of Urology, The First People's Hospital of Suqian, Suqian, China \\ ${ }^{4}$ Department of Urology, ${ }^{\text {nd }}$ Affliated Hospital of Nantong University, \\ Nantong, China \\ ${ }^{5}$ Department of Molecular and Genetic Toxicology, \\ Cancer Center of Nanjing Medical University, Nanjing, China \\ ${ }^{6}$ Department of Urology, \\ First Affiliated Hospital of Nanjing Medical University, \\ Nanjing, China \\ Corresponding author: B. Xu \\ E-mail: njxb1982@126.com
}

Genet. Mol. Res. 13 (4): 10615-10621 (2014)

Received June 11, 2013

Accepted September 19, 2013

Published December 18, 2014

DOI http://dx.doi.org/10.4238/2014.December.18.3

\begin{abstract}
Evidence has shown that miR-146a is involved in carcinogenesis and a common $\mathrm{G} / \mathrm{C}$ variant (rs2910164) in the pre-miR$146 \mathrm{a}$ gene has been found to be associated with various cancers. We investigated the potential prognostic role of miR-146a polymorphism in prostate cancer after radical prostatectomy. Seventy-two southern Chinese with prostate cancer undergoing radical prostatectomy were
\end{abstract}


included in this study. miR-146a polymorphism was analyzed by PCRRFLP. Its prognostic role in biochemical recurrence was assessed using Kaplan-Meier analysis and Cox regression model. We did not find a significant association between miR-146a polymorphism and prostratespecific antigen failure in the Chinese population [HR (95\% (0.30-2.32) for CC vs GG/GC]. However, high Gleason score (over 8) was associated with increased biochemical recurrence and poorer PSAfree survival. This study was limited by the length of follow-up. Future studies with longer follow-up would allow evaluation of more direct metrics, such as disease-specific survival, metastasis-free survival, and overall survival.

Key words: miR-146a; Polymorphism; Prostate cancer; Biochemical recurrence

\section{INTRODUCTION}

Prostate cancer is the most frequently diagnosed malignant disease in males and the second leading cause of cancer deaths in the USA, with more than 78 million new cases occurring globally each year (Siegel et al., 2013). Prostate cancer represents a major public health concern and is associated with significant healthcare costs. The treatment of prostate cancer with surgical resection, which may be combined with hormone therapy, radiation therapy, or chemotherapy, is curative in many patients. However, some patients eventually relapse and develop metastatic disease, which has a poor prognosis because no effective treatments are currently available. Thus, early treatment of patients with high risk of recurrence will be of great help to reduce the mortality of prostate cancer.

MicroRNAs, a class of small noncoding 18-25 nucleotide RNAs, are predicted to regulate expression of almost all protein-encoding genes, thereby affecting diverse cellular and molecular processes at the post-transcriptional level, mainly by binding to the $3^{\prime}$ untranslated region (UTR) of their target mRNAs (Amiel et al., 2012). Prostate cancer progression is associated with altered expression of multiple oncogenes and tumor suppressors, and miRNAs may potentially regulate these genes (Wu et al., 2014). Based on these findings, miRNAs are thought to have a number of potential clinical applications in prostate cancer as biomarkers and therapeutic targets. In our previous study, miR-146a was found downregulated in castration-resistant prostrate cancer tissues, and overexpression of miR-146a in androgenindependent cell lines not only inhibited cell growth, colony formation, and migration in vitro, but also reduced tumorigenicity and angiogenesis in vivo (Xu et al., 2012). Also, the expression of miR-146a in prostate cancer tissues was controlled by a common G/C polymorphism located in the stem-loop of pre-miR-146a (rs2910164). We found that subjects with the CC homozygous genotype had a 0.65 -fold lower risk than those with the GG/GC genotypes, and the $\mathrm{C}$ allele displayed a lower prevalence of prostate cancer compared with the $\mathrm{G}$ allele (Xu et al., 2010). However, the prognostic role of rs 2910164 in prostate cancer has not been reported.

In the present study, we assessed the potential prognostic roles of miR-146a polymorphism on the recurrence of prostrate-specific antigen (PSA) in clinically localized prostate cancer after radical prostatectomy to evaluate the prognostic aspect of rs2910164 in prostate cancer. 


\section{MATERIAL AND METHODS}

\section{Study population}

A total of 72 patients diagnosed with prostate cancer by transrectal prostate biopsy received radical prostatectomy in Zhongda Hospital, Southeast University in Nanjing, China and First Affiliated Hospital of Nanjing Medical University during 2004-2011. All patients were of the Chinese Han population, without androgen deprivation therapy before radical prostatectomy. The cutoff value of postoperative PSA level $\geq 0.2 \mathrm{ng} / \mathrm{mL}$ was considered biochemical recurrence. Peripheral blood was obtained from patients after informed consent was obtained. Research protocols were approved by Zhongda Hospital, Southeast University and First Affiliated Hospital of Nanjing Medical University.

\section{Genotyping}

Polymerase chain reaction and restriction fragment length polymorphism (PCRRFLP) was used to confirm genotypes. PCR was done in a total volume of $10 \mu \mathrm{L}$, containing 5 ng genomic DNA, 2.5 pmol each primer, $4 \mu \mathrm{L}$ ddH2O, and $5 \mu \mathrm{L}$ PCR Mix (Roche Inc.). PCR consisted of 35 thermal cycles at $95^{\circ} \mathrm{C}$ for $30 \mathrm{~s}, 62^{\circ} \mathrm{C}$ for $40 \mathrm{~s}$ and $72^{\circ} \mathrm{C}$ for $45 \mathrm{~s}$, using a PTC 200 Thermal Cycler (Bio-Rad Inc.). Primers were 5'-CATGGGTTGTGTCAGTGTCAGAGCT3' (sense) and 5'-CCTTCAGAGCCTGAGACTCTGCC-3' (antisense). For RFLP analysis, PCR products were digested with SacI $(5 \mathrm{U})$ at $37^{\circ} \mathrm{C}$ overnight, separated on a $3.0 \%$ agarose gel and subsequently stained with ethidium bromide. Two researchers performed RFLP and gel reading and $10 \%$ of the samples were retested, demonstrating that the results were all consistent.

\section{Statistical analysis}

Associations between clinicopathological factors with rs2910164 polymorphism were assessed by the chi-square test or Fischer exact test. The probability of biochemical recurrence-free time was estimated using Kaplan-Meier plots and the log rank test. The Cox regression model was used for multivariate analysis. The Statistical Package of the Social Sciences software version 16.0 (SPSS, Inc.) was used for statistical analyses. A two-sided P value < 0.05 was considered to be statistically significant.

\section{RESULTS}

\section{MiR-146a rs2910164 genotypes and clinicopathological characteristics}

In total, 72 clinical localized prostate cancer patients who underwent radical prostatectomy were included in the final analysis. Among them, 24 (33.3\%) experienced recurrence of PSA during the 24 months follow-up periods. Regarding the association of the clinicopathological features with the genotypes (Table 1), there were no significant differences between age at diagnosis, preoperative PSA level, clinical stage and Gleason scores. 
Table 1. Association between miR-146a polymorphism and clinicopathologic features among post-radical prostatectomy prostate cancer patients $(\mathrm{N}=72)$.

\begin{tabular}{|c|c|c|c|}
\hline & \multicolumn{3}{|c|}{ miR-146a rs2910164 } \\
\hline & $\mathrm{CC}(\mathrm{N}=18)$ & $\mathrm{GG}+\mathrm{CG}(\mathrm{N}=54)$ & $\mathrm{P}$ \\
\hline $\begin{array}{l}\text { Age (year) } \\
{[\text { Means } \pm \text { SD }}\end{array}$ & $68.94 \pm 4.65$ & $67.81 \pm 5.31$ & 0.424 \\
\hline Preoperative PSA $(\mathrm{ng} / \mathrm{mL})$ & & & 0.303 \\
\hline $\begin{array}{l}{[\text { Means } \pm \text { SD }} \\
\text { Pathological stage }\end{array}$ & $15.58 \pm 17.56$ & $22.20 \pm 25.07$ & 0.745 \\
\hline $\begin{array}{l}\text { pT1-2 } \\
\geq \text { pT3 }\end{array}$ & $\begin{array}{r}15(83.3) \\
3(16.7)\end{array}$ & $\begin{array}{l}41(75.9) \\
13(24.1)\end{array}$ & $0.14 \mathrm{~J}$ \\
\hline Surgical margin & & & 0.177 \\
\hline Positive & $12(66.7)$ & $25(46.3)$ & \\
\hline Negative & $6(33.3)$ & $29(53.7)$ & \\
\hline Perineural invasion & & & 0.719 \\
\hline Positive & $2(11.1)$ & $9(16.7)$ & \\
\hline Negative & $16(88.9)$ & $45(83.3)$ & \\
\hline Gleason score & & & 0.163 \\
\hline$\leq 7$ & $17(91.4)$ & $42(77.8)$ & \\
\hline$\overline{8}-10$ & $1(5.6)$ & $12(22.2)$ & \\
\hline
\end{tabular}

\section{Polymorphisms and biochemical recurrence after radical prostatectomy}

Using the Kaplan-Meier method, we compared biochemical recurrence-free time between miR-146a rs2910164 CC genotype and GG+CG genotypes. However, there was no significant correlation $(\mathrm{P}=0.925$, Figure 1$)$.

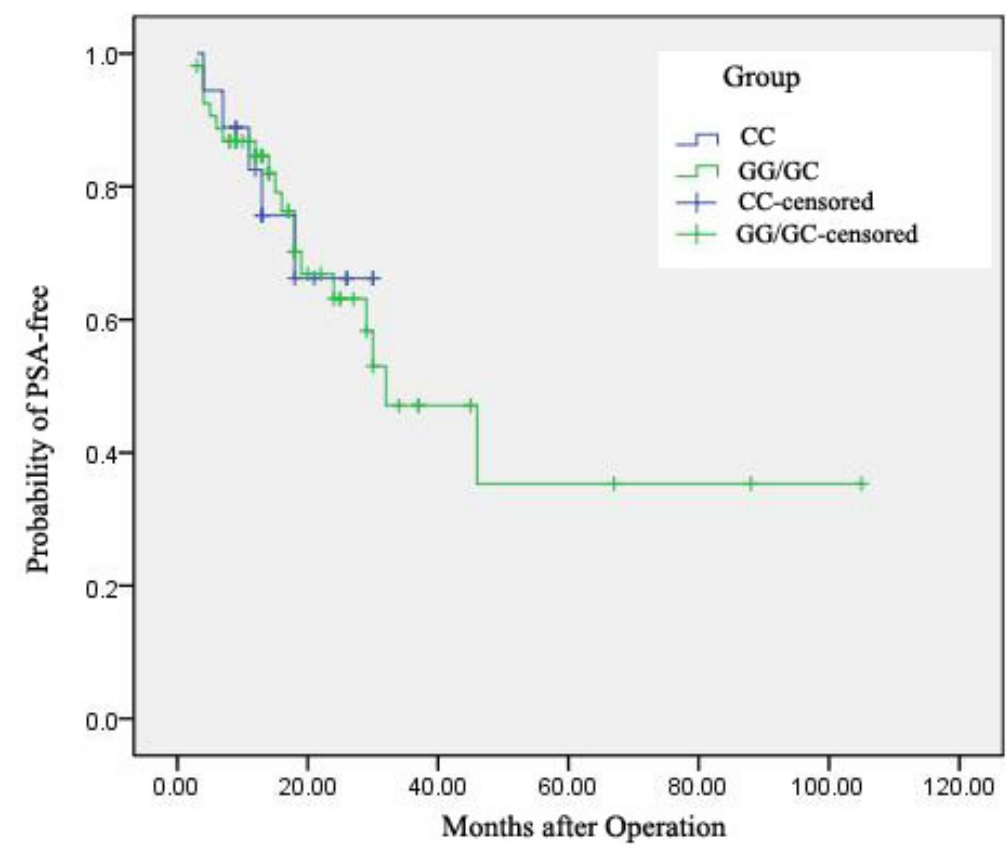

Figure 1. Kaplan-Meier survival curve for biochemical recurrence after radical prostatectomy. 


\section{Multivariate analysis of factors predicting biochemical recurrence after radical prostatectomy}

In the multivariate Cox proportional hazard model, after adjusting for other covariates of age and clinical and pathological factors, Gleason score $\geq 8$ remained an independent protective factor for PSA failure (hazard ratio, 3.61; 95\% confidence interval, 1.01-12.05; $\mathrm{P}=$ 0.036), while miR-146a rs2910164 polymorphism did not show a risk effect on PSA failure $(\mathrm{P}=0.722$, Table 2$)$.

$\begin{aligned} & \text { Table 2. Multivariate Cox proportional hazards analysis of factors predicting PSA recurrence after radical } \\
& \text { prostatectomy. }\end{aligned}$
\begin{tabular}{lcc} 
& \\
\hline Variables & Hazard ratio $(95 \%$ confidence interval $)$ & P \\
\hline Age (year; $>70$ versus $\leq 70)$ & $0.95(0.87-1.03)$ & 0.947 \\
Preoperative PSA $(\mathrm{ng} / \mathrm{mL} ; \geq 10$ versus $<10)$ & $1.00(0.98-1.02)$ & 0.876 \\
Pathological stage $(\geq \mathrm{pT} 3$ versus pT1-2) & $2.56(0.64-10.21)$ & 0.183 \\
Gleason score $(8-10$ versus $\leq 7)$ & $3.61(1.01-12.05)$ & 0.036 \\
Positive surgical margin & $0.83(0.36-1.93)$ & 0.667 \\
Perineural invasion & $1.66(0.46-5.98)$ & 0.437 \\
miR-146a rs2910164 $[\mathrm{CC}$ versus $(\mathrm{GG}+\mathrm{GC})]$ & $0.83(0.30-2.32)$ & 0.722 \\
\hline
\end{tabular}

\section{DISCUSSION}

One-third of prostate cancer cases were at intermediate-risk (i.e., T1-T2; PSA $<20 \mathrm{ng}$ / $\mathrm{mL}$ and $\mathrm{GS}<8$ ) when diagnosed (Beheshti et al., 2013). These patients may exhibit great variability in outcome as 5-year PSA-based biochemical failure rates range from 9 to $44 \%$ following radical prostatectomy (Roberts and Han, 2009). In addition, many PSA recurrent patients are prone to develop metastatic lesions accompanied by significant mortality. Therefore, new markers are needed to accurately predict the risk of relapse and enhance the selection of highrisk patients who will benefit from adjuvant therapy and intensive follow-up.

MicroRNAs, including miR-146a, have been demonstrated to contribute to the complex molecular mechanisms involved in the control of cell growth, differentiation and survival processes, which are mainly related to cancer development and progression (Labbaye and Testa, 2012). MiR-146a has been found to be lost in several cancers such as breast cancer (Bhaumik et al., 2008), pancreatic cancer (Li et al., 2010) and prostate cancer (Lin et al., 2008; $\mathrm{Xu}$ et al., 2012), and its expression reduces the progressive potential of cancer cells by directly targeting BRMS1, IRAK1, TRAF6, ROCK1, and EGFR. Furthermore, we previously found that overexpression of miR-146a inhibited androgen-independent cells proliferation and migration in vitro and reduced tumorigenicity and angiogenesis in vivo by modulating the EGFRERK pathway. Moreover, its expression in prostate cancer tissues was controlled by a common polymorphism in the miR-146a gene, which was also demonstrated to be correlated with cancer susceptibility in various malignancies. In addition, miR-146a and its polymorphism have been found to be predictors of the prognosis of oropharynx cancer (Guan et al., 2013), bladder cancer (Wang et al., 2012), and glioma (Permuth-Wey et al., 2011). However, how miR-146a polymorphism works in prostate cancer prognosis has not yet been reported. In the present study, we attempted for the first time to determine the prognostic role of rs2910164, but we failed to find a positive correlation between this polymorphism and PSA failure of 
prostate cancer $(\mathrm{P}=0.722)$. However, pathological Gleason score remains the most reliable predictor [HR: 3.61 (1.01-12.05), $\mathrm{P}=0.036$ ].

In terms of study limitations, this was a retrospective study, and it covered a wide study period. We did not carry out a blind review of all specimens; however, it must be noted that the pathological evaluation was carried out by two urological pathologists in the entire study period, thus standardizing the Gleason grade evaluation and mitigating the aforementioned drawback. Furthermore, many patients were excluded from the final analysis because of missing data or they were submitted to adjuvant therapy, with possible selection bias. In the present retrospective study, the genotypes of miR-146a polymorphism were not associated with risk of biochemical recurrence (BCR), as well as age, preoperative PSA level, pathological stage, positive surgical margin and perineural invasion. However, Gleason score represented one of the most reliable indicators for the decision of adjuvant treatment after radical prostatectomy.

This study was also limited by the length of follow-up. Future studies with longer follow-up would allow evaluation of more direct metrics, such as disease-specific survival, metastasis-free survival, and overall survival.

\section{Conflicts of interest}

The authors declare no conflict of interest.

\section{ACKNOWLEDGMENTS}

Research supported by National Natural Science Foundation of China (\#81370849, \#81300472, \#81070592 and \#81202034), Natural Science Foundation of Jiangsu Province (\#BL2013032 and \#BK2012336) and Nanjing City (\#201201053) and Southeast University (\#3290002402), and Science Foundation of Ministry of Education of China (\#20120092120071). The funders had no role in study design, data collection and analysis, decision to publish, or preparation of the manuscript.

\section{REFERENCES}

Amiel J, de Pontual L and Henrion-Caude A (2012). miRNA, development and disease. Adv. Genet. 80: 1-36.

Beheshti M, Haim S, Zakavi R, Steinmair M, et al. (2013). Impact of 18F-choline PET/CT in prostate cancer patients with biochemical recurrence: influence of androgen deprivation therapy and correlation with PSA kinetics. J. Nucl. Med. 54: 833-840.

Bhaumik D, Scott GK, Schokrpur S, Patil CK, et al. (2008). Expression of microRNA-146 suppresses NF-kappaB activity with reduction of metastatic potential in breast cancer cells. Oncogene 27: 5643-5647.

Guan X, Sturgis EM, Song X, Liu Z, et al. (2013). Pre-microRNA variants predict HPV16-positive tumors and survival in patients with squamous cell carcinoma of the oropharynx. Cancer Lett. 330: 233-240.

Labbaye C and Testa U (2012). The emerging role of MIR-146A in the control of hematopoiesis, immune function and cancer. J. Hematol. Oncol. 5: 13.

Li Y, Vandenboom TG, Wang Z, Kong D, et al. (2010). miR-146a suppresses invasion of pancreatic cancer cells. Cancer Res. 70: 1486-1495.

Lin SL, Chiang A, Chang D and Ying SY (2008). Loss of mir-146a function in hormone-refractory prostate cancer. $R N A$ 14: 417-424.

Permuth-Wey J, Thompson RC, Burton NL, Olson JJ, et al. (2011). A functional polymorphism in the pre-miR-146a gene is associated with risk and prognosis in adult glioma. J. Neurooncol. 105: 639-646.

Roberts WB and Han M (2009). Clinical significance and treatment of biochemical recurrence after definitive therapy for 
localized prostate cancer. Surg. Oncol. 18: 268-274.

Siegel R, Naishadham D and Jemal A (2013). Cancer statistics, 2013. CA Cancer J. Clin. 63: 11-30.

Wang M, Chu H, Li P, Yuan L, et al. (2012). Genetic variants in miRNAs predict bladder cancer risk and recurrence. Cancer Res. 72: 6173-6182.

Wu K, Liu J, Tseng SF, Gore C, et al. (2014). The role of DAB2IP in androgen receptor activation during prostate cancer progression. Oncogene 33: 1954-1963.

Xu B, Feng NH, Li PC, Tao J, et al. (2010). A functional polymorphism in Pre-miR-146a gene is associated with prostate cancer risk and mature miR-146a expression in vivo. Prostate 70: 467-472.

Xu B, Wang N, Wang X, Tong N, et al. (2012). miR-146a suppresses tumor growth and progression by targeting EGFR pathway and in a p-ERK-dependent manner in castration-resistant prostate cancer. Prostate 72: 1171-1178. 